Version number and date: Version 2---December $9^{\text {th }} 2008---$

\title{
Magnetotransport properties of high-quality cobalt nanowires grown by focused-electron-beam-induced deposition
}

\author{
A. Fernández-Pacheco ${ }^{1,2,3}$, J.M. De Teresa ${ }^{2,3, *}$, R. Córdoba ${ }^{1,3}$, M.R. Ibarra ${ }^{1,2,3}$ \\ ${ }^{1}$ Instituto de Nanociencia de Aragón, Universidad de Zaragoza, Spain \\ ${ }^{2}$ Instituto de Ciencia de Materiales de Aragón, Universidad de Zaragoza-CSIC, Facultad de \\ Ciencias, Zaragoza, 50009, Spain \\ ${ }^{3}$ Departamento de Física de la Materia Condensada, Universidad de Zaragoza, Spain
}

\section{Abstract:}

High-quality cobalt nanowires have been grown by focused-electron-beam-induced deposition (FEBID) and their magnetic and transport properties determined. The nanowires contain up to about $95 \%$ Co atomic percentage, as measured by EDX spectroscopy, which remarkably represents a high value compared to other metal deposits grown by the same technique. The Co content has been found to correlate with the beam energy used for the growth. The magnetotransport properties have been studied on individual nanowires through 4-probe measurements. For the nanowires with the highest Co content, the resistivity at room temperature is low $(\sim 40 \mu \Omega \mathrm{cm})$, and shows metallic temperature dependence. The magnetotransport properties clearly demonstrate the ferromagnetic nature of the nanowire, with a saturation magnetization of $M_{s}=1329 \pm 20 \mathrm{emu} / \mathrm{cm}^{3}$, very close to the bulk one. Due to the local character of this type of growth at targeted places and its high lateral resolution, these results pave the way for the creation of magnetic nanostructures and devices with the full potentiality of high-quality Co.

PACS: 75.47.-m 73.63.-b 81.07.-b 81.15.Jj 
The local deposition of materials using a focused (electron or ion) beam in the presence of a gas precursor is a well-known technique used for the mask-less fabrication of structures with nanometer resolution [1]. This technique is typically employed for the deposition of metals, and has been widely utilized in applications such as the reparation of lithography masks [2], direct fabrication of nanodevices [3], protection for lamella preparation [4], contact of nanowires [5], etc. The possibility of growing nanometric magnetic materials is especially attractive, since it widens the applications to fields such as magnetic storage, magnetic sensing, etc. However, long-standing problems seem to exist for this kind of deposits, mostly associated with the typical organo-metallic nature of the precursor gas. In the case of deposits assisted by a focused electron beam (focusedelectron-beam-induced deposition: FEBID), the low efficiency of the process for decomposing the precursor molecules gives rise to a predominant $\mathrm{C}$-content composition, with low metallic percentages (for instance, for $\mathrm{Pt}$ and $\mathrm{W}$ it is around 15-25\% [6-8]). The abundant amorphous carbon in these cases governs the electrical transport properties, resulting in non-metallic behaviour, with resistivities orders of magnitude larger than the bulk value $[9,10]$. Post-deposition purification techniques are often used to obtain deposits with higher metallic content $[6,11]$, although changes in initial dimensions and creation of voids seem to be unavoidable [11]. The metallic percentages are higher in the case of deposits nanofabricated by a focused ion beam, with resistivities nearer to the bulk value than in FEBID. However, the ion implantation, as well as the high disorder found for these deposits makes them behave far from the desired metallic way [1214].

Regarding the growth of magnetic materials with these techniques, cobalt deposition has been mainly performed [15-21]. Co particles grown by focused ion beam for patterning media [15] present magnetic contrast in MFM images, and measurements of an array of 1000 dots by AGFM give an averaged magnetization saturation of $1000 \mathrm{emu} / \mathrm{cm}^{3}$, a $73 \%$ of the bulk value, with a coercive field of 100 Oe. For FEBID cobalt, previous works [16-21] report a Co concentration from $35 \%$ to $80 \%$ (the rest is composed mainly of carbon, and a residual part of oxygen), and 
resistivities in the range from $160 \mu \Omega \mathrm{cm}$ to $5000 \mu \Omega \mathrm{cm}$, definitely higher than that of pure Co ( 6 $\mu \Omega \mathrm{cm})$. The magnetic nature of FEBID Co has been demonstrated by MFM images, either making images of the deposits themselves, or by coating tips and imaging magnetic elements. A different demonstration was done by Boero et al [18], with the fabrication of nanometric Hall crosses, obtaining significant high sensitivities in the $\mathrm{kHz}$ range. Despite the high resistivity measured (700 $\mu \Omega \mathrm{cm}$ ), the magnetic character of the Hall cross was inferred from the presence of the anomalous Hall effect contribution.

In the present work, we demonstrate the feasibility of high-quality FEBID Co nanowires through magnetotransport measurements of individual nanowires. In certain conditions, the deposits are highly pure (around 90\% of atomic cobalt or higher as determined by EDX) and their resistivity is about $40 \mu \Omega \mathrm{cm}$ at room temperature, with a metallic temperature dependence. The Hall effect shows ordinary and anomalous contributions, with different sign as expected in pure Co samples. The saturation magnetization inferred from such measurements is $1329 \pm 20 \mathrm{emu} / \mathrm{cm}^{3}$, very close to the bulk value. The anisotropic magnetoresistance is $0.65 \%$ at $300 \mathrm{~K}$ and $0.8 \%$ at 2 K. Similar magnetotransport properties have been previously observed in polycrystalline Co thin films, demonstrating the high quality of these FEBID structures, as well as their great potentiality. It must be pointed out that the effect of the intrinsic magnetocrystalline anisotropy is minimized in polycrystalline Co nanowires, in sharp contrast to single-crystalline Co nanowires grown by electrodeposition [22], therefore showing different magnetic behaviour.

The deposits were done in previously micropatterned structures. A $250 \mathrm{~nm}$-thick $\mathrm{SiO}_{2}$ layer is obtained by a combined dry and wet oxidation of a $\mathrm{Si}$ wafer, assuring good isolation from the substrate. Metallic electrodes are realized by lift-off using an $\mathrm{Al}(100 \mathrm{~nm}) / \mathrm{Cr}(5 \mathrm{~nm})$ layer. The Co-based deposits close a $12 \mu \mathrm{m}$ gap (horizontal line in the inset of figure 2). Additional transversal deposits were designed to determine, by conventional 4-probe measurements, the longitudinal and transversal components of the resistivity tensor. The Co nanodeposits were done with a commercial "dual beam" equipment (Nova 200 NanoLab from FEI), using a field-emission 
electron column. For deposition, we use an automatized gas-injection system (GIS) with dicobalt octacarbonyl $\left[\mathrm{Co}_{2}(\mathrm{CO})_{8}\right]$ as the precursor material. The GIS tip is positioned about $150 \mu \mathrm{m}$ away from the region of interest in the $\mathrm{z}$ direction and about $50 \mu \mathrm{m}$ away in the $\mathrm{x} / \mathrm{y}$ direction. The GIS is heated to $27^{\circ} \mathrm{C}$ for sublimation, and a 10 -minute pre-heating period is realized before deposition. The process is performed at room temperature, at a chamber pressure of about $2.5 \times 10^{-6}$ mbar. The structures have been grown with beam energy in the range $5-30 \mathrm{kV}$, and beam current in the range 0.13-9.5 nA. Typical dimensions for the deposits are length=9 $\mu \mathrm{m} \times$ width $=500 \mathrm{~nm} \times$ thickness $=200 \mathrm{~nm}$, with deposition time of the order of a few minutes when using high currents and of the order of one hour when using low currents.

We have performed in-situ energy-dispersive X-ray (EDX) microanalysis of several Co nanodeposits with different combinations of electron-beam energy and current in order to study any correlation between the growth conditions and the nanodeposit composition. A summary of the results is displayed in Table I. The set of results clearly demonstrates that the main parameter governing the nanodeposit content is the beam current. For beam currents above $\sim 2 \mathrm{nA}$, the Co content in atomic percentage is around $90 \%$ or more, whereas for lower currents the Co content is typically around $80 \%$ or less. The rest of constituents of the nanodeposits is C and $\mathrm{O}$. As an example, in figure 1 the $\mathrm{Co}, \mathrm{C}$, and $\mathrm{O}$ content is represented as a function of the beam current in deposits performed at $10 \mathrm{kV}$ beam energy. The Co content increases monotonously from about $80 \%$ for beam current of $0.13 \mathrm{nA}$ to about $95 \%$ for beam current above $2 \mathrm{nA}$.

As far as we know, Co percentages above $90 \%$ have not been reported so far [16-21]. A previous study by Utke et al. also found an increase in the Co content as a function of the beam current, but never exceeded $80 \%$ [20]. It has been argued that beam-induced heating is the main effect responsible for this behaviour [20, 21]. The precursor gas molecule, $\mathrm{Co}_{2}(\mathrm{CO})_{8}$, can be thermally decomposed at relatively low temperature, $60^{\circ} \mathrm{C}[23]$. As a consequence, the use of high current will favour local heating, enhancing the precursor molecule dissociation and the high Co content. It can be pointed out that our electron beam source is a field-emission gun, which will 
likely produce smaller beam spots on the substrate than in the case of thermionic-tungsten-filament sources, employed for example in reference 20. Thus, the electron beam current will be focused on a smaller area, producing larger local heating effects. We put forward that this is the reason for the higher Co content in our nanodeposits compared to previous studies. In the following, we will demonstrate that a high content of $\mathrm{Co}$ is required to obtain high-quality magnetotransport properties.

The relevance of the surface oxidation of these cobalt nanowires for the magnetotransport properties has been evaluated by preparing some samples with a 5-10 nm FEBID Pt capping layer. This small FEBID Pt thickness together with its high resistivity [6, 20], guarantees a high resistance in parallel with the Co lines, assuring no perturbation in the Co conduction, as it was subsequently checked. After several days in air, no changes were found in the resistance of any nanowire (covered or not covered). We expect that only the usual passivation layer at the topmost surface will be present in the non-covered nanowires, without any significant influence on the obtained results.

As an example, we will show hereafter the results obtained on a nanowire grown with a beam energy of $10 \mathrm{kV}$ and a beam current of $2.1 \mathrm{nA}$, thus having a high Co content (around 95\%). A wide range of magnetic field-dependent transport measurements has been performed. These measurements have been done in two different equipments, depending on the geometry of the applied magnetic field with respect to the thin film plane. For fields perpendicular to the thin film plane, a commercial PPMS from Quantum Design was used, with fields up to $9 \mathrm{~T}$ and in a temperature range $2 \mathrm{~K}<\mathrm{T}<300 \mathrm{~K}$. In this set-up, perpendicular magnetoresistance and Hall effect were measured. For fields in the plane, the measurements were done in a closed cycle refrigerator $(25 \mathrm{~K}<\mathrm{T}<300 \mathrm{~K})$ with an electromagnet delivering fields up to $1.1 \mathrm{~T}$. In this case, different geometries between the current and the magnetic field were established, as it will be explained below. 
In figure 2 we can see a typical resistivity-versus-temperature measurement for this nanowire. The behaviour is not, as it is the general rule in FEBID deposits, semiconducting-like, but fully metallic, confirming that the residual amount of carbon in the sample is minimal. The resistivity at room temperature is of the order of $40 \mu \Omega \mathrm{cm}$, only a factor of 7 larger than in bulk polycrystalline cobalt. As will be discussed later, this value of resistivity is just related to the small grain size of the FEBID deposit. Another nanowire grown at $10 \mathrm{kV}$ and 0.54 beam current, with Co content around 92\%, also displays similar resitivity value. These FEBID Co deposits are therefore more conductive than the most conductive ones found in literature. The Residual Resistivity Ratio $\left(\mathrm{RRR}=\rho_{300 \mathrm{~K}} / \rho_{2 \mathrm{~K}}\right)$ is about 1.3 , in agreement with results for polycrystalline cobalt thin films [24].

In figure 3 we show the Hall effect resistivity $\left(\rho_{H}=V_{H} t / I\right)$ for the same nanowire as in figure 2 as a function of field for the maximum and minimum temperature measured. $\rho_{\mathrm{H}}$ increases at low fields until reaching magnetic saturation. For higher fields, only the ordinary part variation $\left(\rho_{\mathrm{OH}}\right)$ is observed, with contrary sign to the anomalous one $\left(\rho_{\mathrm{AH}}\right)$. This behaviour has also been observed in magnetron sputtered polycrystalline cobalt films [24]. The ordinary part is obtained by the standard method of fitting the slope at high fields, whereas the anomalous one is determined by extrapolating to $\mathrm{H}=0$ the fit of $\rho_{\mathrm{OH}}$ (see figure 3). From the different isotherms, we find that the relationship $\rho_{\mathrm{AH}} \propto \rho^{2}$ holds in all cases, revealing an intrinsic origin of the Anomalous Hall effect, as it is expected in this conductivity regime $[25,26]$. From the intersection of the linear regions of the ordinary and the anomalous Hall effect [27] (1.42 T at $300 \mathrm{~K})$ and taking into account the demagnetizing factor for the created geometry, we find a saturation magnetization $M_{s}=1329 \pm 20$ emu $/ \mathrm{cm}^{3}$, corresponding to a $97 \%$ of the bulk Co value. We would like to remark that such high magnetization has never been reported before for Co nanodeposits created by FEBID.

From the ordinary part at $2 \mathrm{~K}$, the density of electrons is calculated to be $\mathrm{n}_{\mathrm{e}}=7 \times 10^{-28} \mathrm{~m}^{-3}$. Using this value, together with the experimental value of the resistivity at low temperature, $\rho(0) \approx 27 \mu \Omega \mathrm{cm}$, one can estimate the mean-free-path as in reference [24]: $1(0)=\hbar \mathrm{k}_{\mathrm{F}} / \mathrm{n}_{\mathrm{e}} \mathrm{e}^{2} \rho(0) \approx 4 \mathrm{~nm}$. 
This value is of the order of the grain size determined by high-resolution-transmission-electron microscopy-images (unpublished results), suggesting that the scattering is mainly produced at the grain boundaries between crystalline grains.

In figure $4, \mathrm{MR}$ measurements at $300 \mathrm{~K}$ are shown with three different geometries: $\mathrm{H}$ perpendicular to the thin film plane (perpendicular geometry: PG), $\mathrm{H}$ in plane and parallel to the current I (longitudinal geometry: LG), $\mathrm{H}$ in plane and perpendicular to I (transversal geometry: TG). The measured MR in the three geometries is in perfect agreement with previous results in Co nanowires fabricated by an EBL process [28]. The magnetization aligns along the nanowire axis due to the strong shape anisotropy (length $\approx 18 \times$ width $\approx 45 \times$ thickness). The differences in the saturation fields for TG and PG are due to the width-thickness ratio $(>1)$. The slight decrease of the MR at high magnetic fields is caused by small misalignments of the wire with $\mathrm{H}$. The different magnetoresistance observed between the LG and any of the others is caused by the anisotropic magnetoresistance, $\mathrm{AMR}=100\left(\rho_{/ /-} \rho_{\perp}\right) / \rho$. The value at $300 \mathrm{~K}$ is positive, around $0.65-0.7 \%$, as previously found in Co nanowires [28] and thin films [29]. Supposing coherent rotation when the field is applied in the PG, the anisotropy constant in this geometry, $\mathrm{K}_{\mathrm{u}}{ }^{\mathrm{PG}}$, can be estimated. From reference 28: $R(B)=R_{\| 1}\left(R_{\mid l}-R_{\perp}\right)\left(M_{s} B / 2 \mu_{0} K_{u}{ }^{P G}\right)^{2}$, where in our case, $R_{\mid l}$ and $R_{\perp}$ are the resistances at zero field and after magnetic saturation in the perpendicular direction, respectively. At $300 \mathrm{~K}$, $\mathrm{K}_{\mathrm{u}}{ }^{\mathrm{PG}}=7.9 \times 10^{5} \mathrm{~J} / \mathrm{m}^{3}$ is obtained. In the $\mathrm{TG}$, however, the dependence is far from being quadratic, and the same analysis cannot be performed. This suggests that the mechanism towards saturation in this configuration is not just by coherent rotation of the magnetization. Further experiments with specific magnetic techniques would be requested to investigate in detail the exact mechanisms of magnetization reversal in these nanowires.

The AMR was further studied as a function of temperature by means of the Planar Hall effect (PHE), in a $45^{\circ}$ configuration between $\mathrm{I}$ and $\mathrm{H}$, where the transversal voltage is maximum [30]. In figure 5a) we can see the transversal resistivity $\rho_{x y}$ in this configuration for several isotherms. From $\rho_{x y}$ and under saturation, the AMR ratios can be calculated as $\operatorname{AMR}(\%)=200 \rho_{x y} / \rho$. 
The AMR slightly increases when lowering temperature, from $0.65 \%$ at $300 \mathrm{~K}$ to $0.8 \%$ at $25 \mathrm{~K}$, in good agreement with EBL-fabricated Co nanowires [28]. Figure 5b) shows in detail $\rho_{x y}$ in a low range of field in two selected isotherms. Abrupt switches occur around 150 Oe, the switching field increasing when temperature diminishes.

Finally, and for the sake of completeness, we will show the results obtained in one nanowire grown at $10 \mathrm{kV}$ and low current, $0.13 \mathrm{nA}$, with cobalt content around $80 \%$. As shown in figure 6, such a low cobalt content precludes the achievement of high-quality magnetotransport properties. The room temperature resistivity value, $10800 \mu \Omega \mathrm{cm}$, is more than 300 times larger than in the case of the nanowires grown under high currents. Besides, the study of the resistivity as a function of temperature indicates a slightly semiconducting behaviour, in sharp contrast to the full metallic behaviour found for the nanowires grown under high currents. The current-versusvoltage measurements performed at $25 \mathrm{~K}$ and $300 \mathrm{~K}$ are linear, indicating an ohmic behaviour. The MR measurements shown in the top inset of figure 6 indicate low MR ratios in the perpendicular and transversal configurations, about $-0.08 \%$, one order of magnitude smaller than those found for the nanowires grown under high currents and those expected in bulk polycrystalline Co. Summarizing, nanodeposits with high Co content are required to obtain high-quality magnetotransport properties.

The possibility of growing magnetic devices locally and with high resolution is a longedfor result. We have convincingly demonstrated that Co nanodeposits of high quality can be grown with the FEBID technique. The high flexibility and resolution of the FEBID technique and its compatibility with other lithography techniques are obvious advantages with respect to multi-step processes involving growth and subsequent patterning. Thus, magnetic dots and nanowires or more complex geometrical shapes based on Co can be grown with the FEBID technique. Applications of these cobalt nanodeposits can be expected in domains such as magnetic storage (dots [15]), magnetic sensing (nanoHall probes [18]), tips for MFM [16], spin-polarised transport in carbon nanotubes [31] or other 1D systems, magnetic logic or storage based on domain walls [32], etc. 
In summary, we have done a complete characterization of the magnetotransport properties of FEBID-Co nanowires as a function of temperature. For nanowires grown under high currents, the data found for resistivity, Hall effect and MR are the typical ones for polycrystalline cobalt, with metallic conduction, and saturation magnetization very close to the bulk value. This is in good agreement with EDX measurements, which give Co atomic percentage higher than $90 \%$ in the deposits. However, nanowires grown under low currents show degraded magnetotransport properties. These results, together with the high flexibility and simplicity of FEBID, evidence that this technique represents a fascinating approach to create magnetic nanostructures based on cobalt, with multiple applications in several domains.

*Corresponding author: J.M. De Teresa: deteresa@ unizar.es

\section{Acknowledgements}

Financial support by Spanish Ministry of Science (through projects MAT2005-05565-C02 and MAT2008-06567-C02 including FEDER funding), and the Aragon Regional Government is acknowledged. 


\section{References}

[1] W. F. Van Dorp, B. Van Someten, C. W. Hagen, and P. Kruit, Nanoletters 5, 1303 (2005)

[2] S. Matsui, and Y. Ochiai, Nanotechnology 7, 247 (1996)

[3] A. De Marco, and J. Melngailis, Solid State Electronics 48, 1833 (2004)

[4] L. A. Giannuzzi, and F. A. Stevie, "Introduction to Focused Ion Beams", Boston (2005)

[5] A. Romano-Rodríguez, and F. Hernández-Ramírez, Microelectron. Eng. 84789 (2007)

[6] R. M. Langford, T. X. Wang, and D. Ozkaya, Microelctron. Eng. 84, 784 (2007)

[7] S. Lipp, L. Frey, C. Lehrer, E. Demm, S. Pauthner, and H. Ryssel, Microelectron. Reliab. 11/12 $1779(1996)$

[8] I. J. Luxmoore, I. M. Ross, A. G. Cullis, P. W. Fry, J. Orr, P. D. Buckle, and J. H. Jefferson, Thin Solid Films 5156791 (2007)

[9] F. Wakaya, Y. Tsukatani, N. Yamakasi, K. Murakami, S. Abo, and M. Takai, J. Phys: Conf. Series 38120 (2006)

[10] L. Rotkina, J.-F. Lin, and J. P. Bird , Appl. Phys. Lett 83, 4426 (2003)

[11] A. Botman, J. J. L. Mulders, R. Weemaes, and S. Mentink, Nanotechnology 17, 3779 (2006)

[12] L. Peñate-Quesada, J. Mitra, and P. Dawson, Nanotechnology 18, 215203 (2007) and references therein.

[13] E. S. Sadki, S. Ooi, and K. Hirata, Appl. Phys. Lett. 85, 6206 (2004)

[14] J.-F. Lin, J. P. Bird, 1. Rotkina, P. A. Bennett, Appl. Phys. Lett 82, 802 (2003)

[15] A. Lapicki, E. Ahmad, T. Suzuki, J. Magn. Mag. Mat. 240, 47 (2002); A. Lapicki, K. Kang, and T. Suzuki, IEEE Trans. Magn. 52589 (2002); Y. Kageyama, A. Lapicki, T. Suzuki, J. Magn. Mag. Mat. 272-276, e1314 (2004)

[16] I. Utke, P. Hoffmann, R. Berger, and L. Scandella, Appl. Phys. Lett. 80, 4792 (2002)

[17] I. Utke, T. Bret, D. Laub, Ph. Buffat, L. Scandella, P.Hoffmann, Microelec. Eng. 73-74, 553 (2004) 
[18] G. Boero, I. Utke, T. Bret, N. Quack, M. Todoroa, S. Mouaziz, P. Kejik, J. Brugger, R. S. Popovic, and P. Hoffmann, Appl. Phys. Lett 86, 042503 (2005)

[19] Y. M. Lau, P. C. Chee, J. T. L. Thong, and V. Ng,, J. Vac. Sci. Technol. A 20, 1295 (2002)

[20] I. Utke, J. Michler, P. Gasser, C. Santschi, D. Laub, M. Cantoni, P.A. Buffat, C. Jiao, and P. Hoffmann, Adv. Eng. Mater. 7, 323 (2005)

[21] W.F. van Dorp and C.W. Hagen, J. Appl. Phys. 104, 081301 (2008); I. Utke, P. Hoffmann, and J. Melngailis, J. Vac. Sci. Technol. B 26, 1197 (2008)

[22] Y. Henri, K. Ounadjela, L. Piraux, S. Dubois, J.-M. George, J.-L Duvail, Eur. Phys. J. B 20, $25(2001)$

[23] G.A. West and K.W. Beeson, Appl. Phys. Lett 53, 740 (1988)

[24] J. Kötzler and W. Gil, Phys. Rev. B 72, 060412(R) (2005)

[25] S. Onoda, N. Sugimoto, and N. Nagaosa, Phys. Rev. Lett. 97, 126602 (2006)

[26] S. Sangiao, L. Morellon, G. Simon, J.M. De Teresa, J.A. Pardo, M.R. Ibarra, Phys. Rev. B, in press

[27] M. Rubinstein, F. J. Rachford, W. W. Fuller, and G. A. Prinz, Phys. Rev. B 37, 8689 (1988)

[28] B. Leven, and G. Dumpich, Phys. Rev. B 71, 064411 (2005); M. Brands, and G. Dumpich, J. Appl. Phys 98, 014309 (2005)

[29] W. Gil, D. Görlitz, M. Horisberger, and J. Kötzler, Phys. Rev. B 72, 134401 (2005)

[30] I.A. Campbell and A. Fert, E.P. Wohlfarth, (Ed.), Ferromagnetic Materials, North-Holland, Amsterdam, vol. 3, 1982, p. 747

[31] K. Tsukagoshi, B.W. Alphenaar, H. Ago, Nature 401, 572 (1999)

[32] D.A. Allwood, G. Xiong, C.C. Faulkner, D. Atkinson, D. Petit, R.P. Cowburn, Science 399, $1688(2005)$ 
TABLE I. A. FERNÁNDEZ-PACHECO ET AL

\begin{tabular}{|c|cccc|}
\hline $\begin{array}{c}\text { Electron energy } \\
(\mathbf{k e V})\end{array}$ & $\begin{array}{c}\text { Beam current } \\
(\mathbf{n A})\end{array}$ & $\begin{array}{c}\text { \% C } \\
\text { (atomic) }\end{array}$ & $\begin{array}{c}\text { \% O } \\
\text { (atomic) }\end{array}$ & $\begin{array}{c}\text { \% Co } \\
\text { (atomic) }\end{array}$ \\
\hline 5 & 1.6 & $13.07 \pm 1.74$ & $3.66 \pm 0.72$ & $83.27 \pm 1.02$ \\
\hline 5 & 0.4 & $15.10 \pm 0.33$ & $2.00 \pm 0.17$ & $82.89 \pm 0.51$ \\
\hline 5 & 6.3 & $7.22 \pm 1.00$ & $1.43 \pm 0.40$ & $91.36 \pm 0.64$ \\
\hline 10 & 0.13 & $8.90 \pm 0.20$ & $10.25 \pm 0.20$ & $80.87 \pm 0.20$ \\
\hline 10 & 0.54 & $5.34 \pm 1.09$ & $2.72 \pm 0.69$ & $91.94 \pm 1.77$ \\
\hline 10 & 2.1 & $3.87 \pm 0.92$ & $1.35 \pm 0.65$ & $94.78 \pm 1.55$ \\
\hline 10 & 8.4 & $5.21 \pm 0.10$ & $1.17 \pm 0.23$ & $93.62 \pm 0.27$ \\
\hline 18 & 9.2 & $9.26 \pm 0.63$ & $2.40 \pm 0.27$ & $88.34 \pm 0.66$ \\
\hline 30 & 0.15 & $19.23 \pm 0.50$ & $19.30 \pm 0.20$ & $61.50 \pm 0.50$ \\
\hline 30 & 9.5 & $3.22 \pm 0.21$ & $0.21 \pm 0.12$ & $96.57 \pm 0.40$ \\
\hline
\end{tabular}




\section{Figure captions}

1. Atomic percentage of Co (blue triangles), $\mathrm{C}$ (black squares), and $\mathrm{O}$ (red dots) as a function of the electron-beam current in nanodeposits grown with the FEBID technique at beam energy of 10 $\mathrm{kV}$ using $\mathrm{Co}_{2}(\mathrm{CO})_{8}$ as the precursor gas. Details are given in the text.

2. Resistivity of a Co nanowire grown at $10 \mathrm{kV}$ beam energy and high current, $2 \mathrm{nA}$, as a function of temperature. Fully metallic behaviour is observed. The inset is a SEM image of one of the created devices. The current flows through the horizontal deposit, whereas transversal deposits are performed for four-probe measurements. Deposits are done on top of a $\mathrm{SiO}_{2}$ substrate in which $\mathrm{Al}$ pads were previously patterned by conventional optical lithography techniques.

3. Hall resistivity at $300 \mathrm{~K}$ and $2 \mathrm{~K}$ in the same Co nanowire shown in figure 2 . The magnetic character is manifested by the presence of the anomalous Hall effect. The ordinary part is determined by the slope at high $\mathrm{H}$, whereas the anomalous constant is calculated by extrapolating this slope at zero field. From the intersection of the linear regions of the ordinary and the anomalous Hall effect the value of $\mathrm{NM}_{\mathrm{S}}\left(\mathrm{N}=\right.$ demagnetising factor, $\mathrm{M}_{\mathrm{S}}=$ saturation magnetization $)$ is obtained, which allows the determination of $\mathrm{M}_{\mathrm{s}}$ (see text for details).

4. Magnetoresistance measurements at $300 \mathrm{~K}$ in longitudinal, transversal and perpendicular geometries of the same Co nanowire as in figures 2 and 3.

5. a) Planar Hall effect resistivity isotherms performed in the same Co nanowire shown in figures 2 to 4 . The magnetic field is applied in the substrate plane, forming $45^{\circ}$ with the current, where the PHE voltage is maximum. From these measurements, the AMR has been determined as a function of temperature (see text for details). b) Detail of a) at low magnetic fields, showing abrupt 
magnetic switches as a consequence of the strong shape anisotropy and the magnetic field configuration. The lines are guides to the eye.

6. Resistivity as a function of temperature of a Co nanowire grown at $10 \mathrm{kV}$ beam energy and low current, $0.13 \mathrm{nA}$. The bottom inset shows the current-versus-voltage measurement at $25 \mathrm{~K}$ and the top inset displays magnetoresistance measurements at the same temperature. The high value of the resistivity, the semiconducting temperature dependence and the low MR values illustrate the degraded magnetotransport properties of nanowires grown at low current. 
FIGURE 1. A. FERNÁNDEZ-PACHECO ET AL

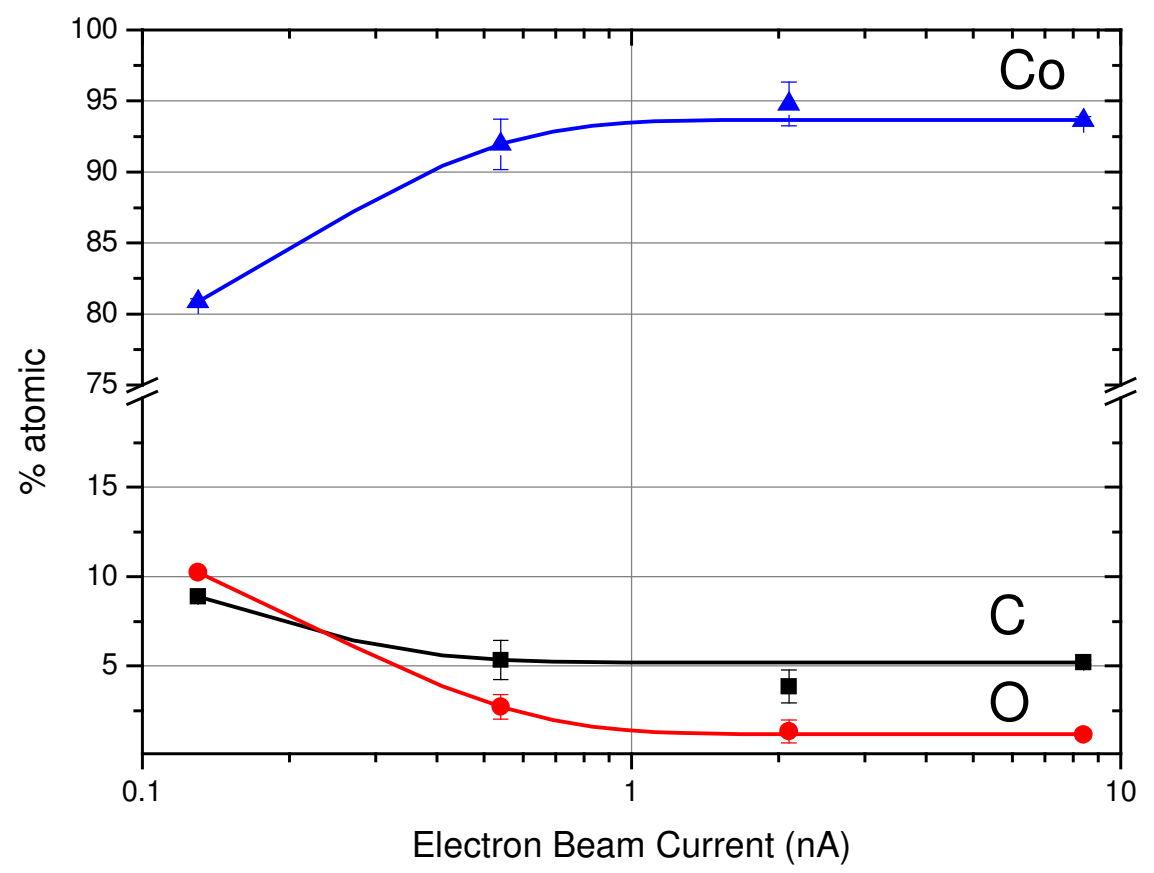


FIGURE 2. A. FERNÁNDEZ-PACHECO ET AL

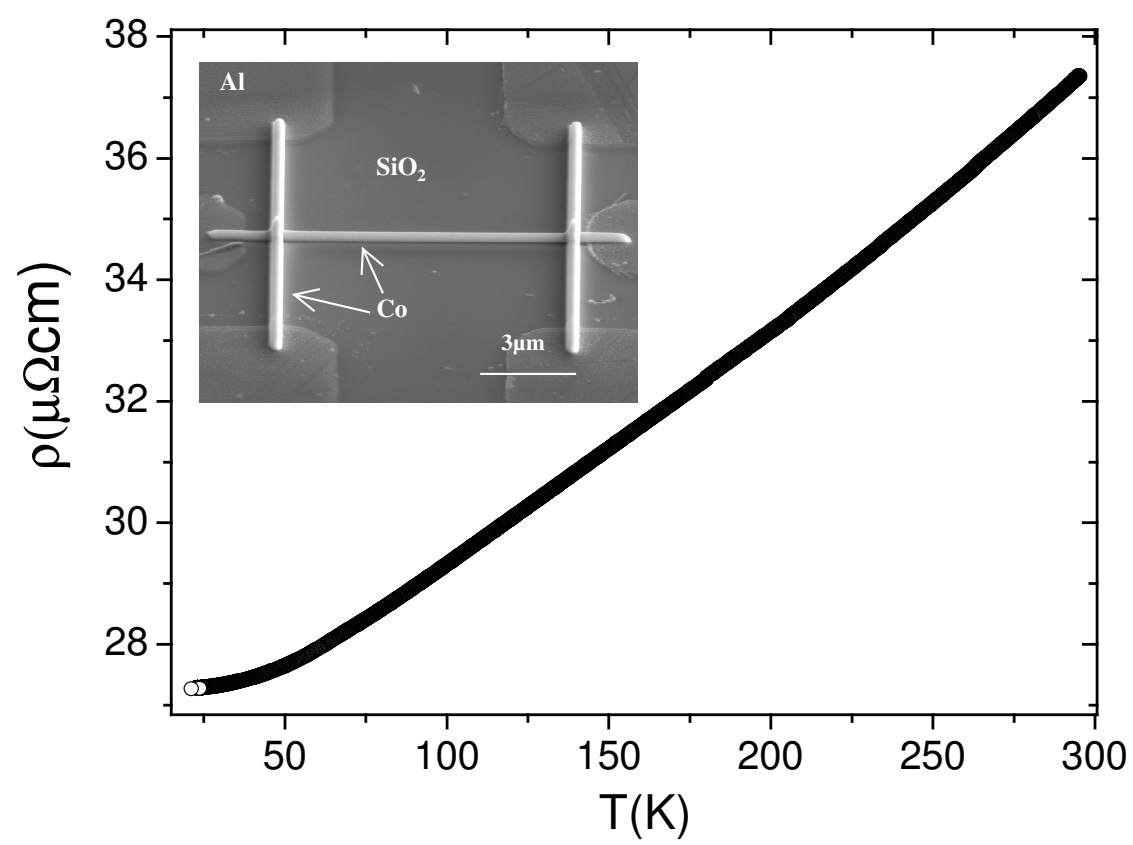


FIGURE 3. A. FERNÁNDEZ-PACHECO ET AL

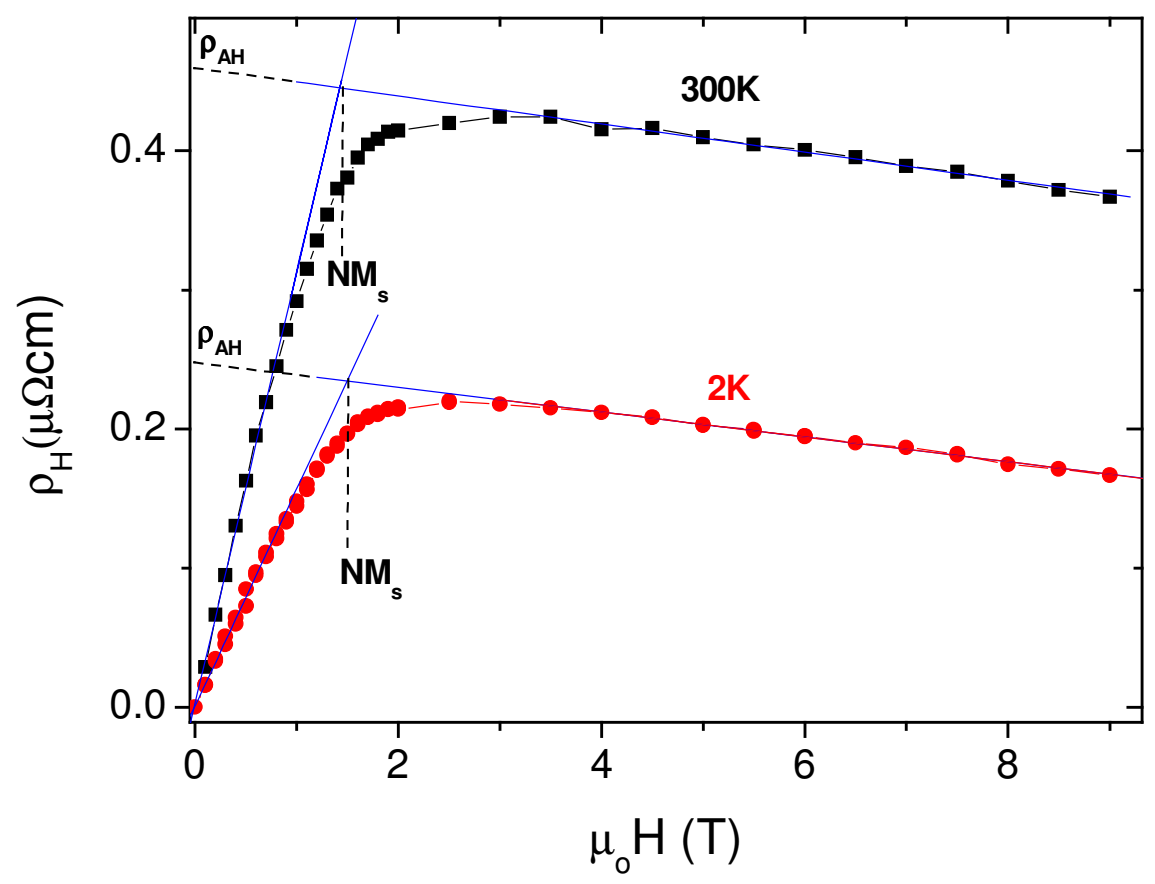


FIGURE 4. A. FERNÁNDEZ-PACHECO ET AL

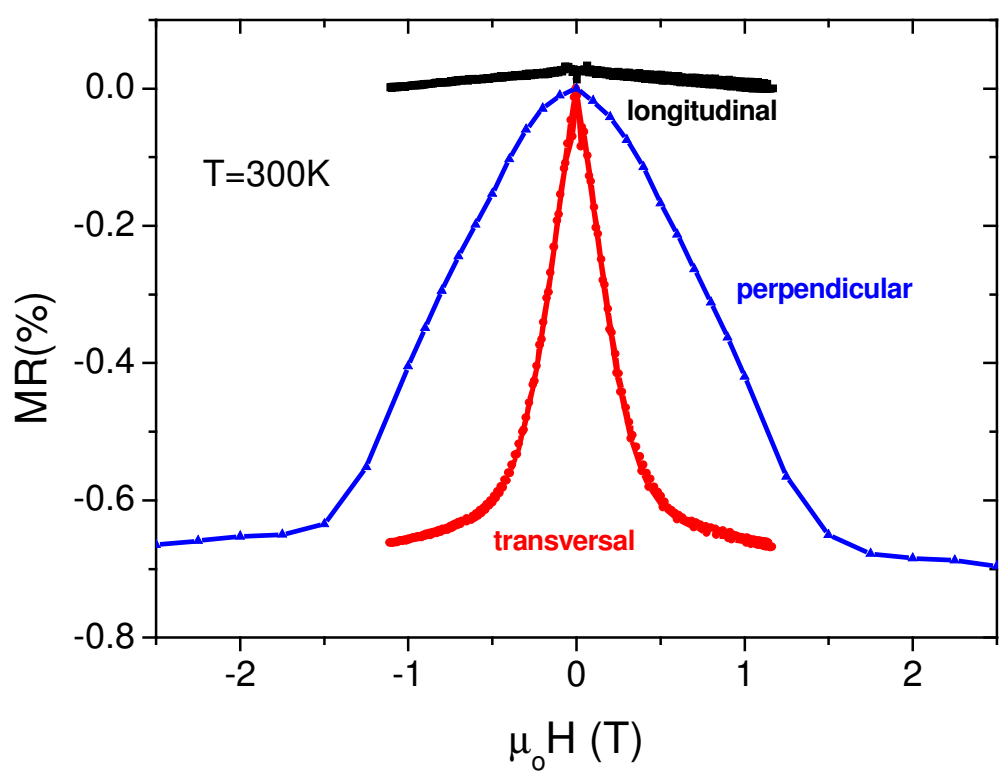


FIGURE 5. A. FERNÁNDEZ-PACHECO ET AL
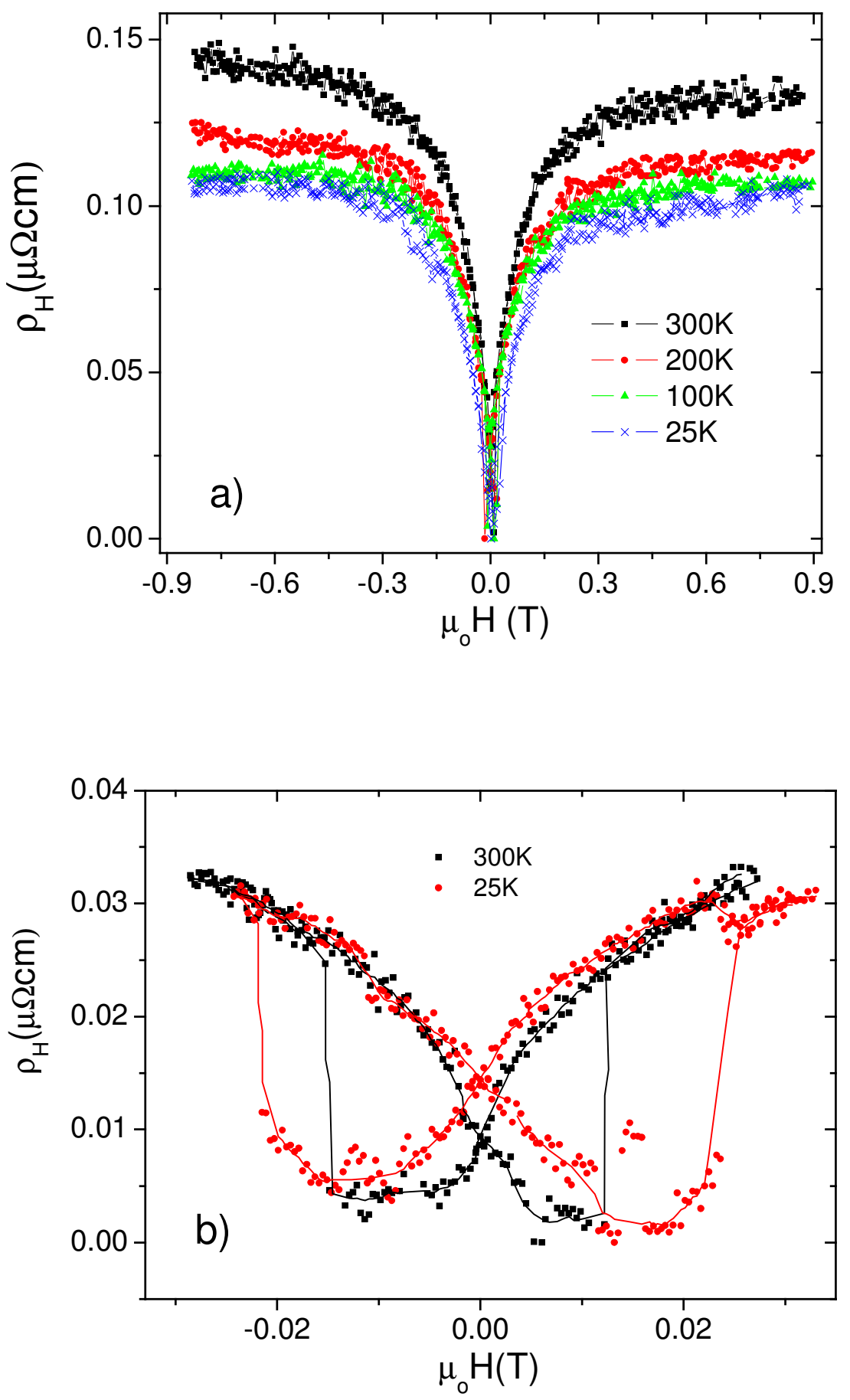
FIGURE 6. A. FERNÁNDEZ-PACHECO ET AL

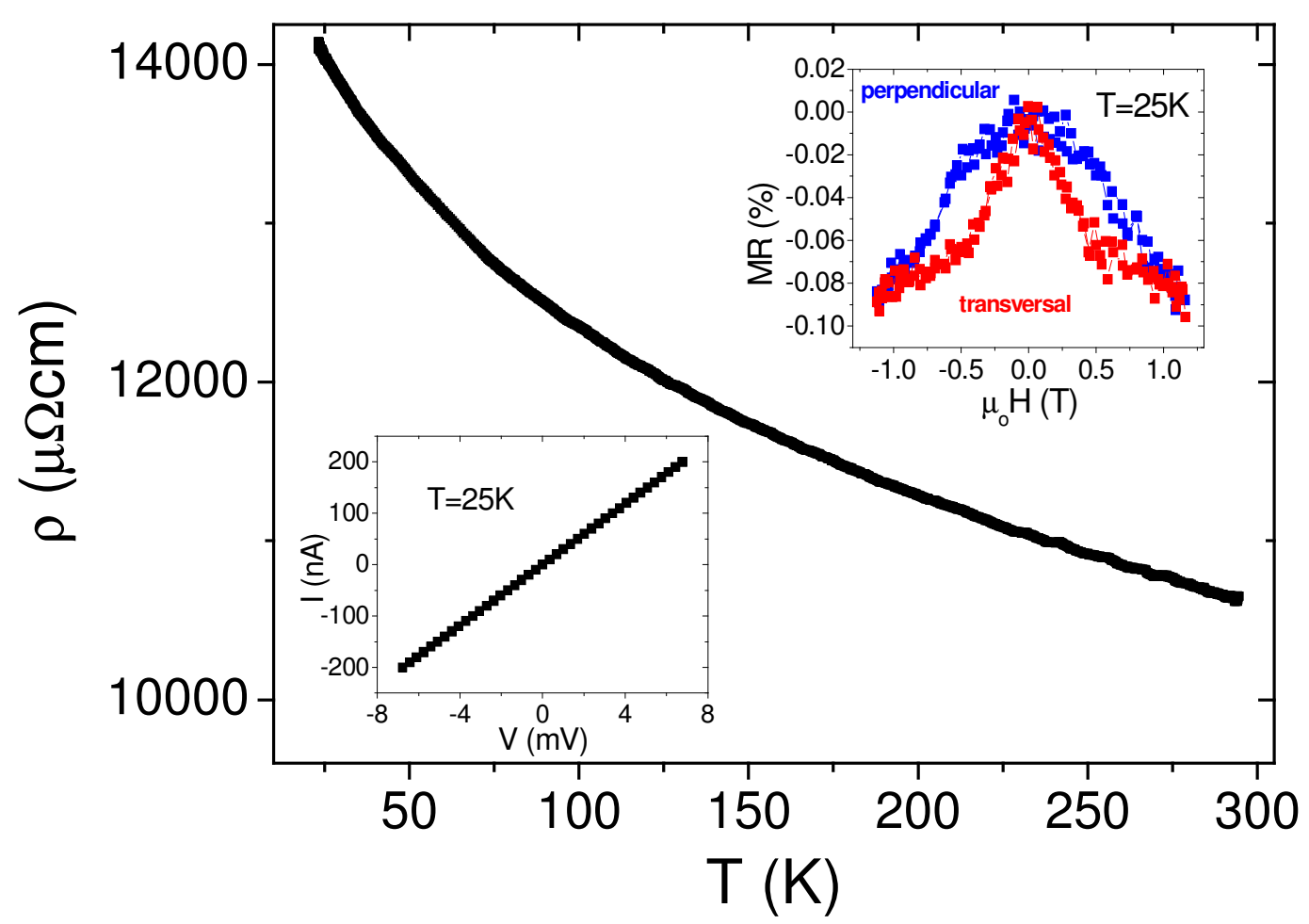

